\title{
The Use of High-Speed Imaging Systems for Applications in Precision Agriculture
}

\author{
Bilal Hijazi ${ }^{1,2}$, Thomas Decourselle2, Sofija Vulgarakis Minov ${ }^{1,2}$, \\ David Nuyttens ${ }^{1}$, Frederic Cointault ${ }^{2}$, Jan Pieters ${ }^{3}$ and Jürgen Vangeyte ${ }^{1}$ \\ ${ }^{1}$ Institute for Agricultural and Fisheries Research (ILVO) \\ ${ }^{2}$ AgroSup Dijon, UP GAP \\ ${ }^{3}$ Faculty of Bioscience Engineering, Ghent University \\ 1,3Belgium \\ ${ }^{2}$ France
}

\section{Introduction}

The evolution of digital cameras and image processing techniques over the last decade has inspired researchers in many fields, particularly agricultural research. Agricultural researchers have used imaging systems in diverse applications, including a multispectral system in viticulture (Hall et al., 2003) and an imaging system to count wheat ears (Cointault et al., 2008).

High speed imaging (HSI) has been widely used for industrial and military applications such as ballistics, hypervelocity impact, car crash studies, fluid mechanics, and others. In agriculture HSI is mainly used in two domains that both require fast processing: fertilization and spraying.

- Fertilization, be it organic or mineral, is essential to agriculture. Over-fertilization can reduce yield and lead to environmental pollution (Mulligan et al., 2006). To prevent these consequences, the fertilization process must be controlled. In Europe and worldwide, mineral fertilization is performed using centrifugal spreaders because they are more cost-efficient than pneumatic spreaders. The process of centrifugal spreading is based on spinning discs which eject large numbers of grains at high speeds (30 to 40 $\left.\mathrm{ms}^{-1}\right)$. To control the spreading process and to predict the distribution pattern on the soil, several characteristics need to be accurately evaluated, i.e., ejection parameters such as velocity and direction, plus granulometry and the angular distribution.

- The spray quality generated by agricultural nozzles plays an important role in the application of plant protection products. The ideal nozzle-pressure combination should maximize spray efficiency by increasing deposition and transfer of a lethal dose to the target (Smith et al., 2000) while minimizing residues (Derksen et al., 2008) and off-target losses such as spray drift (Nuyttens et al., 2007a) and user exposure (Nuyttens et al., 2009a). The most important spray characteristics influencing the efficiency of the pesticide application process are the droplet sizes, the droplet velocities and directions, the volume distribution pattern, the spray sheet structure and length, the structure of 
individual droplets and the 3D spray dimensions. The mechanism of droplets leaving a spray nozzle and their impact on the surface are very complex and difficult to quantify or model. Accurate quantification techniques are therefore crucial.

Without accurate quantification techniques, it is not possible to evaluate the characteristics of the processes in question. Both fertilization and spray processes occur with a relatively high speed. We therefore developed HIS with adequate image processing techniques to characterize the process of centrifugal spreading and the process of pesticide spraying.

This chapter addresses the application of HIS in fertilization and pesticide spraying. To begin, we present the state of the art of characterization methods. A presentation of the devices of acquisition, the applied image processing techniques, and the obtained results follows. We end by discussing these results and present possible future avenues of research.

\section{The state of the art of characterization methods for pesticide spraying and fertilizer centrifugal spreading}

\subsection{Centrifugal spreading}

Persson (1998) evaluated the quality of the spread pattern for different settings by collecting the spread grains in trays. Piron \& Miclet (2006) developed a new concept: the spreader rotates over a radial placed single row of collector trays. Instead of the normal transverse distribution in a cartesian coordinate system, a polar measurement system is used. These methods can be used only for pre-calibration, they are done in test halls and the correct adjustment of the spreader is generally not verified by the farmers.

Grift \& Hofstee (1997) proposed a completely different approach, i.e., a combination of a ballistic model and optical sensors. These sensors determine the initial conditions of flight (velocity, direction) of the particles and their size. Subsequently, the spatial distribution of particles is calculated by introducing the calculated parameters in the ballistic model. This system provides only information for one individual granule and not for the entire flow, however, which makes it inapplicable to real fertilization conditions.

The evolution of digital cameras and imaging techniques have made it possible to surpass the limitations of previous methods. Several new approaches using imaging systems have been investigated (Cointault et al 2003; Vangeyte \& Sonck, 2005; Villette et al. 2007; Bilal et al., 2010, 2011). Villette et al. (2007) developed a method based on blurred images from which the outlet angles of particles can be determined. The angles are introduced in a mechanical model (Olieslagers et al., 1996; Van Liedekerke et al., 2008) to calculate the spread pattern. This method is not yet able to determine all parameters of interest such as granulometry. Cointault \& Vangeyte (2005) used a multi-exposure imaging system that differs in the field of view $\left(1 \mathrm{~m}^{2}\right.$ and $\left.0.01 \mathrm{~m}^{2}\right)$ and in the illumination system used (flashes or LEDs). These systems are very sensitive to noise and are limited by image acquisition conditions (they require a darkened hall to prevent the influence of daylight).

\subsection{Pesticide spraying}

In the past, mainly intrusive methods, also called sampling techniques, were used for spray characterization. With these techniques, droplets were collected and analyzed using 
mechanical sampling devices. However, these sampling devices may affect the spray flow behaviour and can only be used to evaluate spray deposition and estimate droplet size (Rhodes, 1998).

Due to the development of modern technology such as powerful computers and lasers, quantitative optical non-imaging light scattering droplet characterization techniques have been developed for non-intrusive spray characterization. Although these techniques are able to measure some specific spray characteristics, none of them are able to fully characterize a spray application process. Moreover, these techniques are complex, expensive and (in most cases) limited to small measuring volumes. They are not able to accurately measure nonspherical particles. The most important types of non-imaging light scattering droplet characterization techniques are the Phase Doppler Particle Analysers (PDPA) (Nuyttens et al., 2007b, 2009b), the laser diffraction analyzers, e.g., Malvern Analyzer (Stainier et al., 2006), Particle Tracking Velocimetry (PTV), and the optical array probes (Teske et al., 2000). Several studies have shown a wide variation in mean droplet sizes for the same nozzle specifications while using different techniques (Nuyttens, 2007).

The limitations of the non-imaging techniques and the recent improvements in digital image processing, sensitivity of imaging systems and cost reduction, have increased the interest in high-speed imaging techniques for agricultural applications in general, specifically for pesticide applications. Another major advantage is that a visual record of the spray under investigation is available, providing a simple means to verify what is being measured, and perhaps more importantly, what is not being measured (Kashdan et al., 2004 a). Furthermore, another fundamental limitation of light scattering techniques is the inability to accurately measure non-spherical droplets. For this reason, measurements must be obtained sufficiently far downstream from the primary sheet or jet break-up region where ligaments and initially large and often non-spherical droplets are formed. This is an unfortunate limitation, since the near-orifice region is where the process of atomization is occurring and the initial droplets are formed (Kashdan et al., 2004 a).

Recent developments in nozzle technology produce sprays with droplets containing air inclusions. Because these internal structures can cause uncertainty with techniques that rely on diffraction or scattering, interest has been renewed in droplet sizing using imaging techniques. Moreover, imaging techniques offer greater simplicity over light scattering techniques. One of the main issues using imaging techniques not only the need for automated processing routines but also the problem of resolving the depth-of-field (DOF) effect and its inherent influence on measurement accuracy (Kashdan et al. 2004b).

\section{Overview of high-speed imaging used for spraying and spreading}

Generally speaking, high-speed imaging analyzers are spatial sampling techniques consisting of a (strobe) light source, a (high-speed) camera and a computer with image acquisition and processing software. The image frames from the video are analyzed using various image processing algorithms to determine particle (fertilizer grain or spray droplet) characteristics. The imaging techniques have the potential to determine the particles' velocity and other important characteristics like ejection angle and the distribution of the particles. 
Several industrial imaging techniques (PDIA, PIV, LIF) are used for particle characterization. Although these techniques are not applicable to characterize the fertilizer spreading process, they have the potential to fully characterize spray characteristics in a non-intrusive way. For pesticide applications, however, technical and financial challenges make this impossible to put into practice. These techniques are currently mainly used for the characterization of small sprays, e.g., paints, medical applications, fuel injectors, etc.

Some of the available imaging techniques for industrial spray characterization are discussed below (3.1.1 - 3.1.3).

Other interesting techniques were proposed to characterize pesticide sprays and fertilizer spreaders using either a high-speed camera with a high-power light source (3.1.4) or a highresolution standard camera with a strobe light (3.1.5). These techniques can give additional information about the particles' trajectory, which is needed to predict the outcome on the plant (spraying) or in the field (spreading).

\subsection{Imaging techniques}

\subsubsection{Particle/Droplet Imaging Analyzers (PDIA)}

Particle Droplet Imaging Analyzers (PDIA) automatically analyze digital images of a spray (Fig. 1). A very short flash of light illuminates a diffusing screen to back-illuminate the subject. A digital camera with a microscope lens captures images of the subject. Different magnification settings can be used to measure a very wide range of droplet sizes. Image analysis software analyses the images to find drop size. Shape data for the particles can also be measured and recorded. By using dual laser flashes in short succession and measuring the movement of the particle, it is possible to measure the particle velocity. Information on spray geometry can be provided by switching to light sheet illumination. The most common PDIA in use is the Visispray developed by Oxford Laser and is used by Kashdan et al. (2007). This system measures cone angle, drop size and drop velocity and other key parameters of the spray. Kashdan et al. (2004 a; b) made comparisons between the PDIA, PDPA and Laser Diffraction and found good correlation between the results.

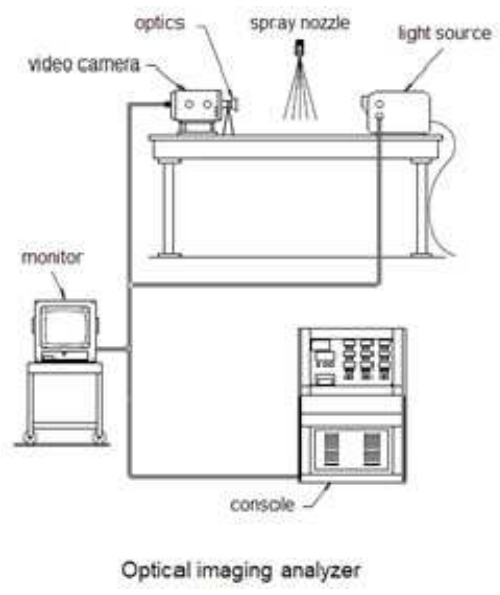

Fig. 1. Typical Particle droplet imaging analyzer (PDIA) (Schick, 1997). 


\subsubsection{Particle Image Velocimetry (PIV)}

Particle Image Velocimetry (PIV) is an optical method used to obtain velocity measurements and related properties of particles. It produces two-dimensional vector fields, whereas other techniques measure the velocity at a point. In PIV, the particle size and density makes it possible to identify individual particles in an image, but not with enough certainty to track it between images. This technique uses laser light and it is well adapted to laboratory conditions but cannot be used in the field. It is rather used as a reference method and not for pesticide spray characterization under practical conditions. Particle Tracking Velocimetry (PTV) (Hatem, 1997) is a variant which is more appropriate with low seeding density experiments, and Laser Speckler Velocimetry (LSV) with high seeding density. Like PIV, PTV and LSV measure instantaneous flow fields by recording images of suspended seeding particles at successive instants in time. Hence, LSV, PTV and PIV are essentially the same technique, but are used with different seeding densities of particles (Paul et al., 2004).

\subsubsection{Laser Induced Fluorescence (LIF)}

Laser Induced Fluorescence (LIF) is a spectroscopic method used to study the structure of molecules, detect selective species, and to perform flow visualization and measurements (Cloeter et al., 2010). The particles to be examined are excited with a laser. The excited particles will, after a few nanoseconds to microseconds, de-excite and emit light at a wavelength larger than the excitation wavelength. This light (fluorescence) is then measured. One advantage that LIF has over absorption spectroscopy is that LIF can produce two- and three-dimensional images, as fluorescence takes place in all directions (i.e., the fluorescence signal is isotropic). By following the movement of the dye spot using high speed camera and image processing, the particle velocity can be determined (Mavros, 2001). LIF can minimize the effect of multiple scattering found with laser diffraction analysers and can minimize the interference between the reflection and refraction lights (Hill \& Inaba, 1989). The drawback of this method is that the particles reflect the LIF signal of the tracers, which can cause error in the measurement signal of the liquid flow.

\subsubsection{High-speed camera with high-power light source}

An alternative method to analyse spray/spreading characteristics is to use a high-speed camera combining high resolution images with a high frame rate. Because of the short exposure time inherent to high-speed imaging, very high illumination intensities are needed. The advantage of this system is the possibility to be adapted to the application condition, the frame rate and the resolution of the image.

Vangeyte et al. (2004) used a high-speed camera (MotionXtra HG 100K, 1504x1128 pixels and frame rate of 1000 images/s) to make a comparison with a multi-exposure imaging system for determination of the trajectories of fertilizer grain ejected from a centrifugal spreader. However, the field of view was small $\left(10 \times 10 \mathrm{~cm}^{2}\right)$. To characterize the full process, all the ejected grains need to be visualized.

Massinon and Lebeau( 2011) used a high-speed camera (Y4 CMOS, Integrated Design Tools) with a high magnification lens (12 x zoom Navitar, $341 \mathrm{~mm}$ working distance) coupled with high-power LED lighting and image processing to study droplet impact and spray retention of a real spray application. Camera resolution was reduced to $1016 \times 185$ pixels to acquire 
20000 images per second with a spatial resolution of $10.58 \mu \mathrm{m}$.pixel-1. A background correction was performed with Motion Studio embedded camera software to get a homogeneous image. Nineteen-LED backlighting (Integrated Design Tools) with a beam angle of $12.5^{\circ}$ was placed $0.50 \mathrm{~m}$ behind the focus area to provide high illumination and a uniform background to the images. Based on the pixel size of the droplet as determined manually from the pictures with Motion Studio software, together with the spatial resolution, the diameter of the droplets was calculated. Similarly, droplet velocities were calculated in a very-time consuming and visual way, based on the distance between the position of the droplet between two consecutive frames and the frame rate. In this way, only the 2-dimensional velocity was calculated.

Many others, like Šikalo et al. (2005) also studied the impact of droplets with a high-speed CCD camera but in these studies, single droplets were produced using a microdrop generator in an on-demand or continuous mode.

\subsubsection{High-resolution standard camera with a strobe light}

This technique combines a high resolution standard (slow speed) camera with a strobe light for tracking high-speed particles. The principle is that a series of light flashes is triggered one after the other over a single camera exposure. The number of flashes determines the maximum number of particle positions that can be recorded on each image.

Cointault et al.(2002 proposed a system combining a monochrome camera (1008x1018 pixels) with a strobe light consisting of photograph flashes to determine the trajectories and velocities of the spread grains in a field of view of $1 \mathrm{mx} 1 \mathrm{~m}$. Vangeyte and Sonck (2005) also used a similar system but with a LED stroboscope and a small field of view $(0.1 \mathrm{~m} \times 0.1 \mathrm{~m})$ to capture the grain flow.

This technique was already used by Reichard et al. (1998) to analyse single droplet behaviour combining a monochrome video camera (60 fields per second) with a single backlight stroboscope (Type 1538-A, Genrad, Concord, MA 01742) at a flash rate of about seven times the field-sequential rate used to drive the camera. This produced multiple images of the same droplet.

Lad et al. (2011) used a high-intensity pulsed laser $(200 \mathrm{~mJ}, 532 \mathrm{~nm})$ as a backlight source which was synchronized with a firewire type of digital camera $(1280 \times 960$ pixels $)$ to analyze a spray atomizer. The laser beam was converted to a laser cone using a concave lens, and then it was diffused by a diffuser. A $200 \mathrm{~mm}$ micro-lens equipped with a spacer was used to get a magnification of 2.6 of the image resulting in a field of view of $1.82 \times 1.36 \mathrm{~mm}$ for a working distance of $250 \mathrm{~mm}$. The digital camera captured shadow images which were analyzed to determine droplet sizes. The system is capable of performing an online characterization of spray droplets and an image calibration was performed using graph paper. A calibration method of an imaging system in the diameter range 4 to $72 \mu \mathrm{m}$ has been reported by Kim and Kim, (1994).

Malot and Blaisot (2000) developed a particle sizing method based on incoherent backlight images using a stroboscope with two fibers synchronized with two cameras. This technique was used to project 2D images of drops on a video camera, which led to two-dimensional images. 


\subsection{Adopted solution}

In both domains (fertilization and spraying), events are relatively fast; typical speeds are 1 to $15 \mathrm{~ms}^{-1}$ in spraying and 30 to $40 \mathrm{~ms}^{-1}$ in fertilization. High-speed cameras with frame rate between 500 and 1000 images per second are needed to capture movement of the particles. However, the size and transparency of the particles are different between the two applications.

- The fertiliser grains are opaque and their diameters are between 3 and $6 \mathrm{~mm}$

- The spraying droplets are translucent and their diameters are between $10-1000 \mu \mathrm{m}$

These differences between the physical characteristics of the particles thus require different setups.

- In fertilization a front-light is adequate and a lens with a focal between 16 and $28 \mathrm{~mm}$ is sufficient.

- Illumination of translucent spray droplets with a front-light is not practical. Hence back-light is used. Because of the small droplet size, a macro lens with a high focal length should be used.

\section{Imaging device and results}

\subsection{Fertilization application}

The aim is to determine the spatial fertilizer distribution on the ground by calculating the ballistics of the particles from their initial conditions of flight (velocity, direction), their properties and geometrical parameters (topography, height and tilt of the discs, etc.). To determine the velocities and the trajectories of the grain at the ejection, imaging devices combined with a image processing techniques can be used. Given that the grains are ejected with a speed of $30-40 \mathrm{~ms}^{-1}$, a HSI system at a minimum rate of 500 images per second is used to film at least the same scene in two different instants. The resulting frame of the same scene is used to estimate the motion of the fertilizer grains.

The fertiliser grains are actually ejected in an arc (Fig 2). To ensure the filming of the same arc, the HSI system has to visualise a field of view of $1 \times 1 \mathrm{~m}^{2}$.

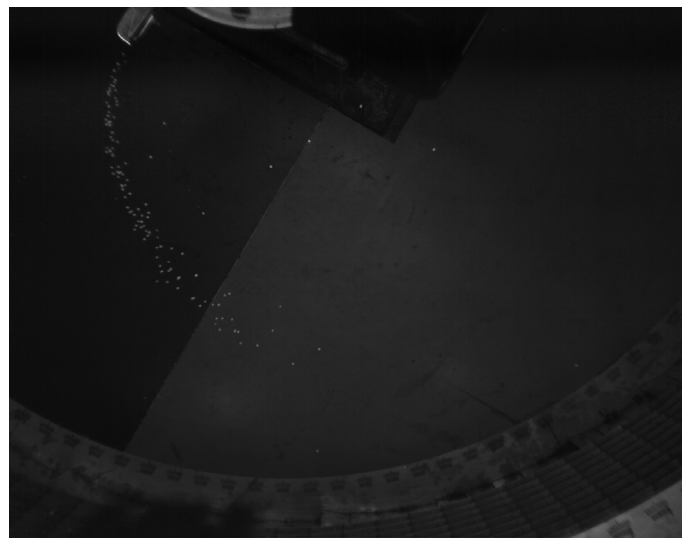

Fig. 2. Image of ejected fertiliser grain. 
Therefore, our system consists of a high-speed camera with a frame rate of $1000 \mathrm{~Hz}$, a sensor of $1280 \times 1042$ pixels ${ }^{2}$, a pixel size of $12 \mu \mathrm{m}$ and a lens with $28 \mathrm{~mm}$ focal length. The camera is placed two meters above the field of work.

After image acquisition, the image must processed. During this essential phase, the velocities and the trajectories must be predicted in order to determine the spatial distribution of the fertiliser grain on the ground (Fig. 3). We have therefore investigated several motion estimation techniques in order to achieve high accuracy.

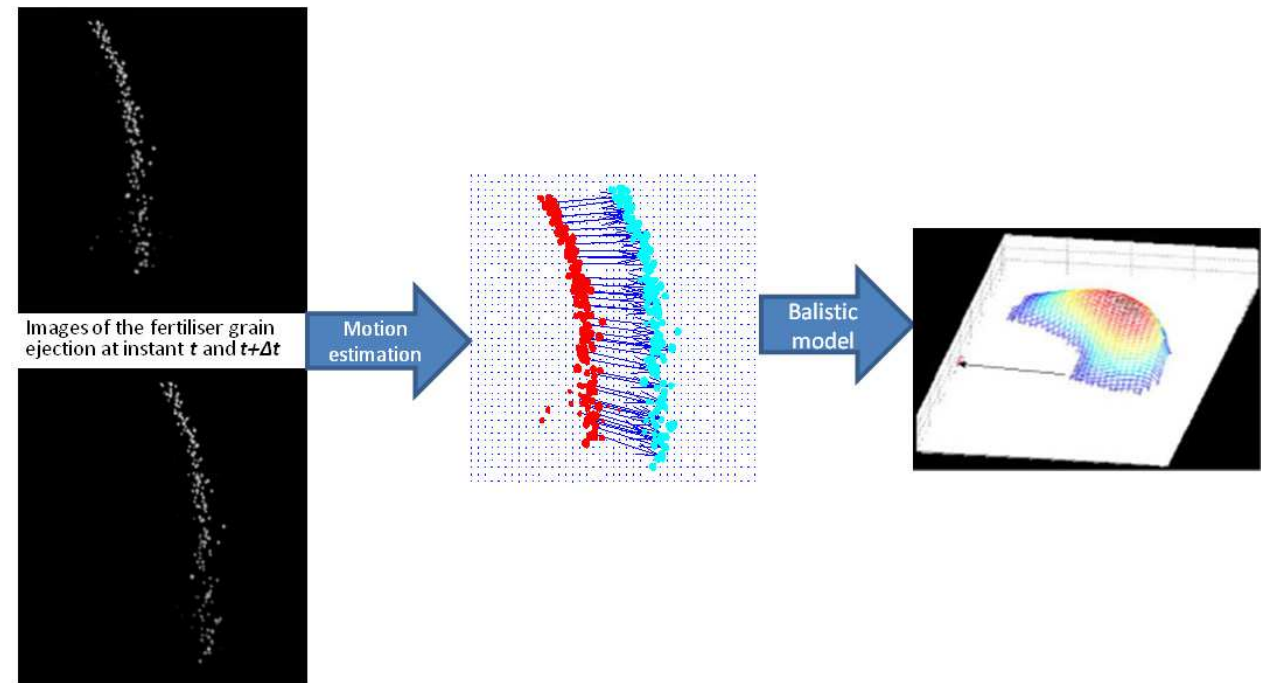

Fig. 3. The images on the left are images of fertilizer grain ejection at the instant $t$ and $\Delta t$; the middle image shows the displacement vector determined by the motion estimation algorithm, and the right image shows the spread pattern determined from the ballistic model.

Barron et al. (1994) divided the optical flow method into four categories: (1) differential methods, (2) region-based matching, (3) energy-based techniques and (4) phase-based techniques. The difference between these methods is the way to resolve the image constraint equation (1):

$$
I(x, y, t)=I(x+d x \Delta t, y+d y \Delta t, t+\Delta t)
$$

I is the intensity of pixels and $\mathrm{dx}$ and $\mathrm{dy}$ are the displacement after $\Delta \mathrm{t}$ (for more details see (Barron et al., 1994)).

The fertilizer grain displacements in pixels/image are very large compared to the displacements generally estimated with classical motion estimation methods. These displacements can therefore not be estimated directly using methods such as Markov Random Fields or optical flow measurement; the maximum displacement detectable by these methods is too small to detect the fertilizer granules' path. Therefore, a theoretical model of the movement of the grains was first combined with a Markov Random Fields method to estimate the motion of the grains on high speed images of the grain flow. This technique had a good accuracy but it was not sufficient to have a very accurate prediction of the spatial distribution. An improved method was needed. 
We then investigated whether Block Matching or motion estimation methods based on Gabor filters could improve the accuracy and eliminate the modeling and minimization steps of the MRF technique.

Although the block matching techniques are able to detect large displacements between different frames, our experiment using block matching techniques showed that it is not suitable for our application (Hijazi et al., 2008). These techniques only give good results when scenes are highly textured, which is not the case for the fertilizer images. In reality, the fertiliser grains have all a similar shape. The probability of erroneous estimation is therefore too high.

For the motion estimation method based on Gabor filters, Spinei's method (Spinei et al., 1998), a triad of controlled Gabor filters was implemented. To expand the range of detectable displacements, this method uses a multi-resolution representation of image sequences. The higher level has a lower resolution. When the resolution is decreased, the displacement decreases with the same ratio. We showed, however, that this method did not improve the accuracy on the measurement of the displacements (Hijazi et al., 2008).

Because of the similarity between the fertiliser grain images and the images used in PIV to study the turbulence phenomena in fluid, it is possible to apply the proven high-accuracy PIV algorithms to estimate the movement of the fertilizer granules.

A two-step cross correlation algorithm with sub-pixel accuracy for motion estimation was applied to the fertilizer granules' motion during centrifugal spreading. In this method, the first step is to fit an arc of a circle in the grain region of each image (Fig. 3). These arcs are used to divide the grain region in several smaller regions. For each region, a global motion displacement is then determined. The second step uses the global displacement to determine the local displacement using a normalized cross-correlation. The final results, with their subpixel accuracy, created the possibility to develop a system based on a low-resolution camera sensor. For more details about the techniques see Hijazi et al., 2010, 2011.

A comparison with the result of the MRF technique clearly shows that the crosscorrelation method determines very precisely the fertiliser granule velocities with an average error of 0.4 pixel or less, and $90 \%$ of the granule velocity with a rate of error less than 0.2 pixel (Table 1$)$.

\begin{tabular}{|c|c|c|c|c|}
\hline \multirow{2}{*}{} & \multicolumn{2}{|c|}{ Cross-correlation } & \multicolumn{2}{c|}{ MRF } \\
\cline { 2 - 5 } & horizontal & vertical & horizontal & vertical \\
\hline $\begin{array}{c}\text { Mean velocity } \\
\text { modulus (pixel) }\end{array}$ & \multicolumn{2}{|c|}{62.402} & 0.453 \\
\hline Bias error (pixel) & 0.085365 & 0.099817 & 1.624881 & 0.800443 \\
\hline $\begin{array}{c}\text { Error maximum } \\
\text { (pixel) }\end{array}$ & 0.384418 & 0.330194 & 5.549145 & 3.431636 \\
\hline $\begin{array}{c}\text { Standard } \\
\text { deviation (pixel) }\end{array}$ & 0.073746 & 0.080768 & 1.399179 & 0.834144 \\
\hline $\begin{array}{c}\text { Accuracy 90\% } \\
\text { (pixel) }\end{array}$ & 0.17261 & 0.21957 & 3.65780 & 2.34400 \\
\hline
\end{tabular}

Table 1. Comparison between the cross correlation method and the MRF method. 


\subsection{Spray application process}

In a precision spraying context, the analysis of droplet behaviour on the leaves (adhesion, bounce or splash) and the link with leaf surface features, particularly its roughness, is one of the most important steps. Our study features two main parts. One aspect is to analyze the surface and to extract features using texture analysis methods. This characterizes the leaf roughness. The other aspect is to analyze the droplet and its behaviour using HSI and associate image processing techniques. This chapter only discusses the analysis of the droplet and its behavior. We use a system composed of a high-speed camera with a highpower light source and a droplet generator (Figs. 4 and 5).

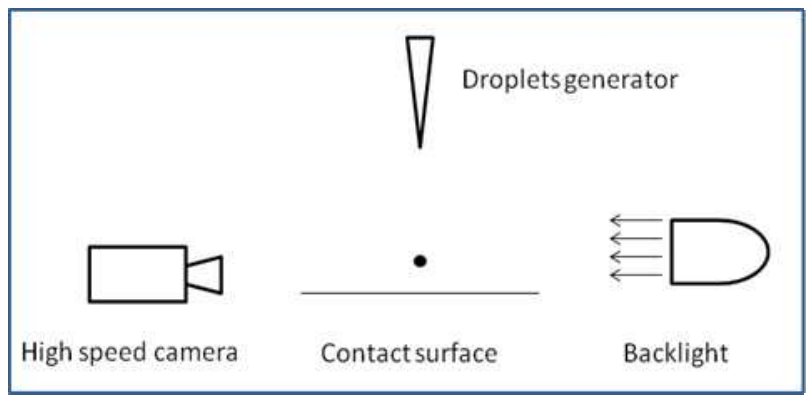

Fig. 4. Scheme of the system for single spray droplet characterization.

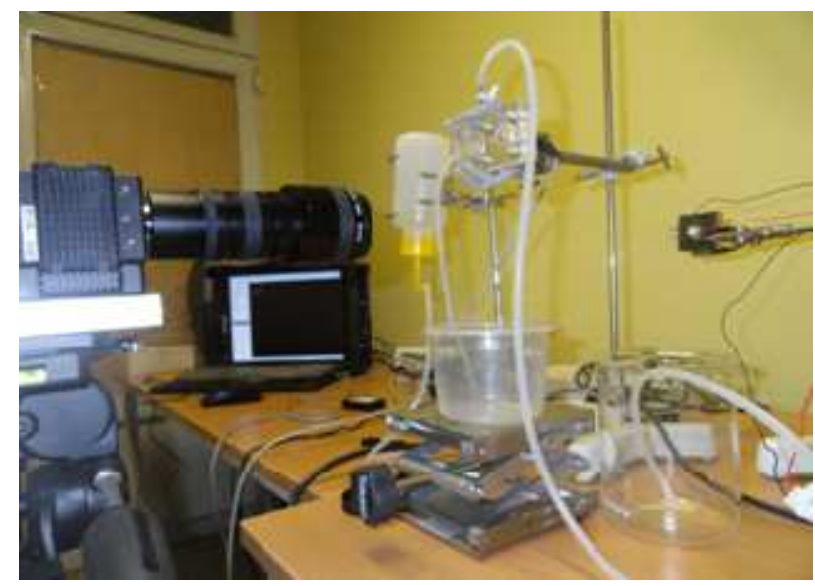

Fig. 5. Picture of the system.

The droplet generator runs in "on demand" mode and creates single droplets. Depending on its features (size, velocity, surface, composition), a droplet can have different behaviours after impact such as adhesion, bounce or shatter. We influence the size and velocity of the droplet by using several nozzles and changing the height of fall of the droplet.

The small size of the droplets $(80-400 \mu \mathrm{m})$ requires use of a macro lens with a high focal length. In addition to these constraints, we have to set up the camera with a high frame rate (1000 frames/s) and a low exposure time $(16 \mu \mathrm{s})$ in order to extract accurate information of 
size, velocity and behaviour of the droplets. Consequently we illuminate the scene with a LED system which provides high illumination and uniform background that leads in wellcontrasted images for easier tracking of the droplets.

Object tracking is an important task within the field of computer vision. Computer performance has increased and high-quality cameras are now available for a reasonable price. These advancements have led to increased interest in object tracking algorithms. Video analysis has three key steps: (1) detection of moving objects of interest, (2) tracking such objects from frame to frame, and (3) analyzing object tracks to recognize their behaviour (Yilmaz et al., 2006).

The first task is to define a suitable representation of the object. The object can be represented in several ways, such as points, primitive geometric shapes or object contours. The point is the simplest representation. The point representation is not suitable here because we need to extract the size of the droplet from the video. A circular shape as primitive geometric shape for droplet representation could be a good solution in order to extract the size, but it may lead to wrong interpretation of the behaviour of the droplet because it may be hard to distinguish adhesion from bounce. We therefore use a contour representation for the droplet.

The next task is to determine the way to detect the object. Almost all tracking algorithms require detection of the objects either in the first frame or in every frame. Objects can be detected in the video in different ways. For instance, we can use point detector algorithms to find interest points in images. This method is well adapted for images with expressive texture in localities, but this is not the case of our images. Another way could be to use segmentation methodsn but this can lead to detection errors after impact, when the droplet merges with the contact surface. To overcome these difficulties, we used background subtraction. We acquire a first image corresponding to the background when the droplet is out of the field of view. Then we subtract the background from next images that contain the droplet. Finally techniques of supervised learning could have been used to detect objects and correctly separate surface from droplets but we reject them because the learning step is too time-consuming

We first perform an inversion of the image to get high intensity values for the pixels belonging to the droplet. Then we apply the background subtraction, which allows us to detect only moving objects in the scene. We now track these objects from frame to frame (Fig. 6). To do so, we use a combination of two methods: shape matching and contour tracking.

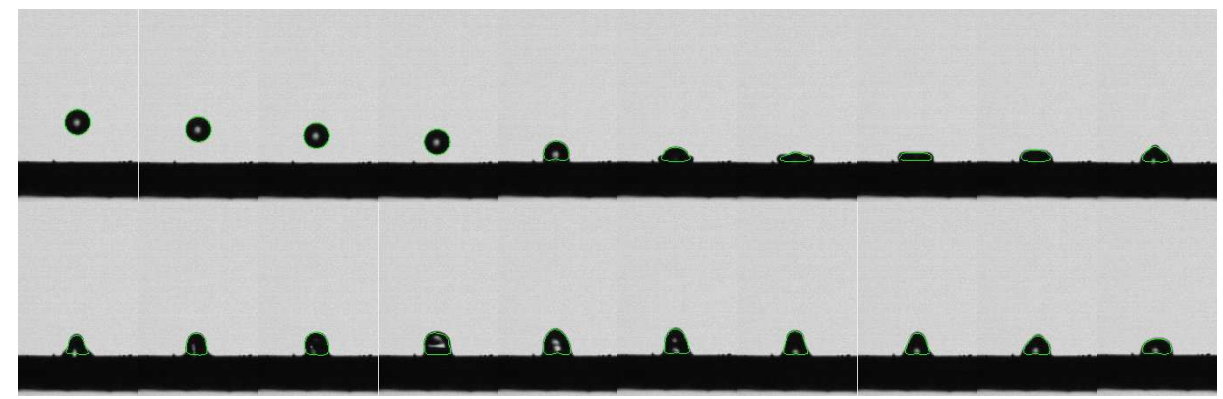

Fig. 6. Sequence of droplet impact with adhesion. 
We consider two main stages in the video, i.e., the time before impact and after impact. Before impact, we use an algorithm of shape matching, because that the droplet keeps a circular shape (Fig. 7). We compute an area-perimeter ratio I defined as:

$$
\mathrm{I}=\frac{4 \pi \mathrm{A}}{\mathrm{P}^{2}}
$$

with $\mathrm{A}$ : area of the object, $\mathrm{P}$ : perimeter of the object.

If $\mathrm{I}$ is equal to 1 , the object has a circular shape and we can consider it as a droplet. We include a tolerance of $5 \%$ for I in order to take into account small deformations of the droplet.

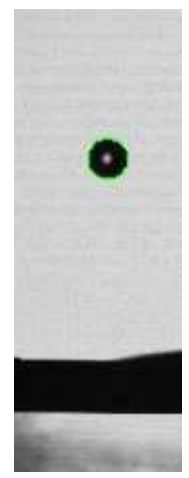

Fig. 7. Droplet detection using shape matching.

Once the droplet reaches the surface, it is subject to bigger deformations during the steps of spreading and recoiling. It is no longer possible to use shape matching for tracking the droplet. We use contour tracking technique named Active Contour, also known as the snake method. The development of active contour models results from the work of Kass et al. (1988). A snake is an active (moving) contour, in which the points are attracted by edges and other image boundaries. To keep the contour smooth, a membrane and thin plate energy is used as contour regularization. Basically, snakes are trying to match a deformable model to an image by means of energy minimization (Fig. 8). The energy functional which is minimized is a weighted combination of internal and external forces. The internal forces emanate from the shape of the snake, while the external forces come from the image and/or from higher level image understanding processes. The snake is parametrically defined as $v(s)=(x(s), y(s))$, where $x(s), y(s)$ are $x, y$ coordinates along the contour and $s$ is from $[1,0]$. The energy functional relative to the snake is written:

$$
E_{\text {snake }}=\int_{0}^{1} E_{\text {int }}(v(s))+E_{\text {image }}(v(s))+E_{\text {con }}(v(s)) d s
$$

- $\mathrm{E}_{\text {int }}$ : internal energy due to bending which serves to impose piecewise smoothness constraint. 
- $\mathrm{E}_{\text {image }}$ : image forces pushing the snake toward image features (edges, lines, terminations).

- $\quad \mathrm{E}_{\mathrm{con}}$ : external constraints are responsible for putting the snake near the desired local minimum

The internal spline energy can be written:

$$
E_{\text {int }}=\alpha(s)\left|\frac{d v}{d s}\right|^{2}+\beta(s)\left|\frac{d^{2} v}{d s^{2}}\right|^{2}
$$

where $\alpha(s), \beta(s)$ specify the elasticity and stiffness of the snake, respectively.

The second term of the energy integral is derived from the image data over which the snake lies. A weighted combination of three different functionals is presented which attracts the snake to lines, edges, and terminations:

$$
E_{\text {image }}=w_{\text {line }} E_{\text {line }}+w_{\text {edge }} E_{\text {edge }}+w_{\text {term }} E_{\text {term }}
$$

The line-based functional may be very simple:

$$
E_{\text {line }}=f(x, y)
$$

where $f(x, y)$ denotes image gray levels at image location $(x, y)$. The sign of $w_{\text {line }}$ specifies wether the snake is attracted to light or dark lines.

The edge-based functional attracts the snake to contours with large image gradients, i.e., to locations of strong edges:

$$
E_{\text {edge }}=-|\operatorname{grad} f(x, y)|^{2}
$$

Line terminations and corners may influence the snake using a weighted energy functional $E_{\text {term }}$. Let $C(x, y)=\left(G_{\sigma}(x, y) * f(x, y)\right)^{2}$ be a smoothed image, with $G_{\sigma}$ a Gaussian with a standard deviation $\sigma$. Let $\theta=\tan ^{-1}\left(\frac{C_{y}}{C_{x}}\right)$ the gradient angle, $n=(\cos \theta, \sin \theta)$ unit vector along gradient, $n_{\perp}=(-\sin \theta, \cos \theta)$ perpendicular to gradient. $E_{\text {term }}$ is defined using curvature of level lines in $C(x, y)$ :

$$
E_{\text {term }}=\frac{\delta \theta}{\delta n_{\perp}}
$$

The snake behaviour is controlled by adjusting the weights $w_{\text {line }}, w_{\text {edge }}$ and $w_{\text {term }}$.

For the moment, only $E_{\text {int }}$ and $E_{\text {image }}$ are used to define the energy of our snake. In order to improve the process of energy minimization, i.e., to reduce the number of iterations in the process of minimization, we plan to create a third energy based on a priori knowledge about the deformation of the droplet. 


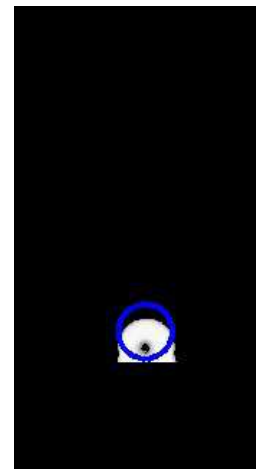

(a)

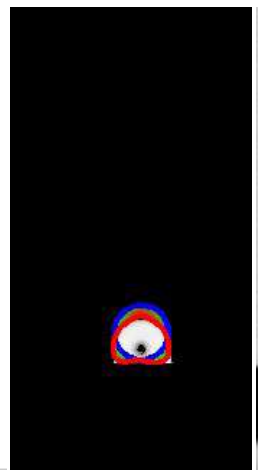

(c)

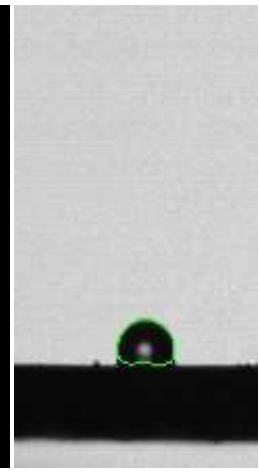

(d)

Fig. 8. (a) Previous contour displayed in image after inversion and background subtraction. (b) Image representing external energy. (c) Image displaying snake evolution. (d) Current contour displayed in original image.

Our tracking methods allows to extract information about size and velocity of the droplet and then calculate the Weber number, $W e$, which is a dimensionless number characterizing a droplet. We is the ratio between kinetic energy and surface energy (Richard \& Quéré, 2000):

$$
W e=\frac{\rho D_{0} v^{2}}{\sigma}
$$

with $\rho$ : density of liquid, $D_{0}$ : diameter of the spherical droplet, $v$ : velocity of the droplet and $\sigma$ : surface tension of the liquid.

More than extracting droplet's features, our tracking method can automatically determine the behaviour of the droplet. For the moment, our algorithm only recognizes adhesion or bounce. In future improvements are planned in order to manage other behaviours as splashing or runoff.

\section{Conclusion}

The aim of this chapter is to show the potential of using high-speed imaging systems in precision agriculture. Here, we present pesticide spraying and fertiliser spreading to illustrate agricultural applications that where HSI can be used to characterise their processes. In centrifugal fertilizer spreading, we developed a HSI device based on a highspeed camera and a high-power light. The images are taken at a frame rate of 1000 images/s. Then a newly developed image processing algorithm is used to determine the grain velocities and trajectories necessary for the characterization of the centrifugal spreading.

In pesticide spraying, we used a HSI system based on a high-speed camera and a back-light system based on power LEDs to determine the pesticide droplet impact. The captured images are used in a tracking algorithm that determines the behaviour of the droplet on the impact surface. 
The results obtained in both applications were promising. More work is needed to fully characterize the processes such as the determination of the granulometry of fertilizer grain, displacement of pesticide droplets in a real spraying process, and the combination of the calculated spray characteristics with leaf roughness.

Only two applications of HSI in agriculture were presented here. However, this technique could be used in other areas of agriculture, such as harvesting, where a fast process needs to be visualised or characterized.

\section{Acknowledgment}

Special thanks go to Mrs Miriam Levenson from ILVO for her help in reviewing this article.

\section{References}

Barron, J. L. \& Thacker, N. A. (2005). Tutorial: Computing 2D and 3D optical flow. In Imaging science and biomedical engineering division .pp 1-12. Manchester: Medical School, University of Manchester. http:/ / www.tina-vision.net/docs/memos/2004012.pdf.

Cloeter, M. D.; Qin, K.; Patil, P. \& Smith, B. (2010). Planar Laser Induced Fluorescence (PLIF) Flow Visualization applied to Agricultural Spray Nozzles with Sheet Disintegration; Influence of an Oil-in-Water Emulsion. ILASS-Americas 22nd Annual Conf. on Liquid Atomization and Spray Systems. Cincinnati, USA, May 2010.

Cointault, F.; Guérin, D.; Guillemin, J.P. \& Chopinet, B. (2008). In-Field Wheat ears Counting Using Color-Texture Image Analysis. Journal of Crop and Horticultural Science, Vol. 36,pp. 117-130.

Cointault, F.; Paindavoine, M. \& Sarrazin, P. (2002). Fast imaging system for particle projection analysis: application to fertilizer centrifugal spreading. Journal Measurement Science and Technology, Vol. 13, pp. 1087-1093.

Cointault, F.; Sarrazin, P., \& Paindavoine, M. (2003). Measurement of fertilizer granules motion on a centrifugal spreader with a fast imaging system. Precision Agriculture, Vol. 4, pp. 279-295.

Cointault, F., \& Vangeyte, J. (2005). Development of low cost high speed photographic imaging systems to measure outlet velocity of fertilizer granules during spreading. In International fertiliser society meeting, Proceeding 555, London, UK. Available from: http://www.fertiliser-society.org/Proceedings/US/Prc555.HTM.

Derksen R. C.; Zhu, H.; Ozkan, H. E.; Hammond, R. B.; Dorrance, A. E. \& Spongberg, A. L. (2008). Determining the influence of spray quality, nozzle type, spray volume, and air assisted application strategies on deposition of pesticides in soybean canopy. Transactions of the ASABE, Vol. 51, No. 5, pp. 1529-1537.

Grift, T. E. \& Hofstee, J. W. (1997). Measurement of velocity and diameter of individual fertilizer particles by an optical method. Journal of Agricultural Engineering Research, Vol. 66, No. 3, pp. 235-238.

Hall, A.; Louis, J. \& Lamb, D.(2003) Characterising and mapping vineyard canopy using high-spatial-resolution aerial multispectral images. Computers and Geosciences, Vol. 29,pp. 813-822. 
Hatem, A. B. (1997). Software development for particle tracking velocimetry. University of Nottingham, United Kingdom.

Hijazi, B.; Cointault, F.; Yang, F. \& Paindavoine, M. (2008). High Speed Motion Estimation of Fertilizer Granules with Gabor Filters. In H. Kleine \& M. Guille'n (Eds.), 28th International congress on high speed imaging and photonics proceedings, SPIE, Canberra, Australia.

Hijazi, B.; Cointault, F.; Dubois, J.; Coudert, S.; Vangeyte, J.; Pieters, J. \& Paindavoine, M. (2010). Multi-phase cross-correlation method for motion estimation of fertiliser granules during centrifugal spreading. Precision agriculture, Vol. 11, No. 6, pp. 684702

Hijazi, B.; Vangeyte, J.; Cointault, F.; Dubois, J.; Coudert, S.; Paindavoine, M. \& Pieters, J. (2011), "Two-step cross correlation-based algorithm for motion estimation applied to fertilizer granules' motion during centrifugal spreading," Optical Engineering. Vol. 50, No. 6, pp. 067002.

Hill, B. D. \& Inaba, D. J. 1989. Use of water-sensitive paper to monitor the deposition of aerially applied insecticides. Journal of Economic Entomology, Vol. 82, No. 3, pp. 974980.

Kashdan, J. T.; Shrimpton, J. S. \& Whybrew, A. (2004 a). Two-phase characterization by automated digital image analysis. Part 2: Application of PDIA for sizing sprays. Particle \& Particle Systems Characterization, Vol. 21, No. 1, pp. 15-23.

Kashdan, J. T., Shrimpton, J. S. \& Whybrew, A. (2004 b). Two-phase characterization by automated digital image analysis. Part 1: Fundamental principles and calibration of the technique. Particle E Particle Systems Characterization, Vol. 20, No. 6, pp. 387-397.

Kashdan, J. T.; Shrimpton, J. S. \& Whybrew, A. (2007). A digital image analysis technique for quantitative characterization of high-speed sprays. Optical Laser Engineering, Vol. 45, pp. 106-115.

Kass, M.; Witkin, A. \& Terzopoulos, D. (1988). Snakes: Active contour models. International journal of computer vision, Vol. 1, No. 4, pp.321_331.

Kim, K. S. \& Kim, S. S. (1994). Drop sizing and depth-of-field correction in TV imaging. Atomization and Sprays. Vol. 4, pp. 65-78.

Lad, N.; Aroussi, A \& Muhamad, M. F. S. (2011). Droplet size measurement for Liquid Spray using Digital Image Analysis Technique Lad. Journal of Applied Sciences, Vol. 11, No. 11, pp. 1966-1972.

Mavros, P. (2001). Flow visualization in stirred vessels. Trans IChemE, Vol. 79, Part A.

Mulligan, D.; Bouraoui, F.; Grizzetti, B.; Aloe, A. \& Dusart, J. (2006). An atlas of PanEuropean data for investigating the fate of agrochemicals in terrestrial ecosystems. Available from: http://www.environmentalexpert.com/sign_in.asp?vienede=http://www.enviro nmental-expert.com/articleemailformbd_login.asp?cid=27957\&codi=26379.

Nuyttens, D. (2007). Drift from field crop sprayers: The influence of spray application technology determined using indirect and direct drift assessment means. PhD thesis $n r$. 772, Katholieke Universiteit Leuven. 293 pp. ISBN 978-90-8826-039-1.

Nuyttens, D.; De Schampheleire, M.; Baetens, K. \& Sonck, B. (2007a). The influence of operator controlled variables on spray drift from field crop sprayers. Transactions of the ASABE, Vol. 50, No. 4, pp. 1129-1140. 
Nuyttens, D.; Baetens, K.; De Schampheleire, M. \& Sonck B. (2007b). Effect of nozzle type, size and pressure on spray droplet characteristics. Biosystems Engineering. Vol. 97, No. 3, pp. 333-345.

Nuyttens, D.; Braekman, P.; Windey, S. \& Sonck, B. (2009a). Potential dermal pesticide exposure affected by greenhouse spray application technique. Pest Management Science, Vol. 65, No. 7, pp. 781-790.

Nuyttens, D.; De Schampheleire, M.; Verboven, P., Brusselman, E. \& Dekeyser, D. (2009b). Droplet size-velocity characteristics of agricultural sprays. Transactions of the $A S A B E$, Vol. 52, No. 5, pp. 1471- 1480.

Olieslagers, R.; Ramon, H. \& De Baerdemaeker, J.(1996). Calculation of Fertilizer Distribution Patterns from a Spinning Disc Spreader by means of a Simulation Model. Journal of Agricultural Engineering Research Vol. 63, No.2, pp. 137-152.

Van Liedekerke, P.; Piron, E.; Vangeyte, J.; Villette, S.; Ramon, H. \& Tijskens, E. (2008). Recent results of experimentation and DEM modeling of centrifugal fertilizer spreading. Granular Matter Vol.10, pp. 247-255.

Paul, E. L.; Atiemo-Obeng, V. A. \& Kresta, S. M. (2004). Handbook of Industrial Mixing: Science and Practice. John Wiley \& Sons, INC., Publication.

Persson, K. \& Skovsgaard, H. (1998). Fertiliser characteristics and spreading patterns from centrifugal spreaders. Proceedings of the International Conference on Agricultural Engineering, AgEng, Oslo, Norway, Paper No. 98-A-058

Piron, E. \& Miclet, D 2006. Spatial distribution measurement: a new method for the evaluation and testing of centrifugal spreaders. In: Proceedings Second International Symposium on Centrifugal Fertiliser Spreading, Cemagref, Montoldre, France, October 24-25, 2006

Reichard, L. D.; Cooper, J. A.; Bukovac, M. J. \& Fox, R. D. (1998). Using a Videographic system to Assess Spray Droplet Impaction and Reflection from Leaf and Artificial Surfaces. Pesticide Science, Vol. 53, pp. 291-299.

Rhodes, M. (1998). Introduction to Particle Technology. John Wiley and Sons Inc.. New Jersey, USA.

Richard, D. \& Quéré, D. (2000). Bouncing water drops. Europhysics Letters (EPL), 50:769_775.

Schick, R. (1997). An engineer's practical guide to drop size. Spraying Systems Co. Wheaton, Illinois, USA.

Šikalo, Š.; Wilhelm, H. D.; Roisman, I. V.; Jakirlić, S. \& Tropea, C. (2005). Dynamic contact angle of spreading droplets: Experiments and simulations. Physics of Fluids, Vol.17, No. 6, pp. 062103.

Smith, D. B.; Askew S. D.; Morris, W. H. \& Boyette, M. (2000). Droplet size and leaf morphology effects on pesticide spray deposition. Transactions of the ASAE, Vol. 43 No. 2,pp. 255-259.

Spinei, A.; Pellerin, D. \& Herault, J. (1998). Spatiotemporal energy-based method for velocity estimation, Signal processing, Vol. 65, pp. 347-362.

Stainier, C.; Destain, M. F.; Schiffers, B. \& Lebeau, F. (2006 a). Droplet size spectra and drift effect of two phenmedipham formulations and four adjuvant mixtures. Crop Protection. Vol. 25, pp. 1238-1243.

Teske, M. E.; Thistle, H. W. \& Hewitt, A. J. (2000). Conversion of droplet size distributions from PMS optical array probe to Malvern laser diffraction. Proceedings ICLASS 2000, Pasadena, CA, USA. 
Vangeyte, J. \& Sonck, B. (2005). Image analysis of particle trajectories. In B. Tijskens, \& H. Ramon (Eds.), Proceedings of the 1st international symposium on centrifugal fertiliser spreading. KULeuven: Leuven, Belgium.

Vangeyte, J. ; Sonck B.; Van Liedekerke, P. \& Ramon, H. (2004). Comparison of two methods to measure the outlet velocity of fertilizer grains from a rotary disc. Proceedings of AgEng 200 edited by the Technology institute, pp. 366-337.

Villette, S.; Cointault, F.; Piron, E.;Chopinet, B. \& Paindavoine, M. (2007). A simple imaging system to measure velocity and improve the quality of fertilizer spreading in agriculture. Journal of Electronic imaging, Vol. 17, No.3, pp. 1109-1119.

Yilmaz, A.; Javed, O. \& Shah, M. (2006) Object tracking: A survey. Acm Computing Surveys (CSUR), Vol. 38, No. 4, pp.13. 


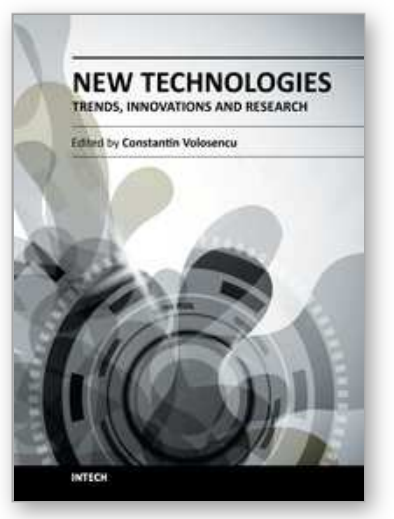

\author{
New Technologies - Trends, Innovations and Research \\ Edited by Prof. Constantin Volosencu
}

ISBN 978-953-51-0480-3

Hard cover, 396 pages

Publisher InTech

Published online 30, March, 2012

Published in print edition March, 2012

The book "New Technologies - Trends, Innovations and Research" presents contributions made by researchers from the entire world and from some modern fields of technology, serving as a valuable tool for scientists, researchers, graduate students and professionals. Some practical applications in particular areas are presented, offering the capability to solve problems resulted from economic needs and to perform specific functions. The book will make possible for scientists and engineers to get familiar with the ideas from researchers from some modern fields of activity. It will provide interesting examples of practical applications of knowledge, assist in the designing process, as well as bring changes to their research areas. A collection of techniques, that combine scientific resources, is provided to make necessary products with the desired quality criteria. Strong mathematical and scientific concepts were used in the applications. They meet the requirements of utility, usability and safety. Technological applications presented in the book have appropriate functions and they may be exploited with competitive advantages. The book has 17 chapters, covering the following subjects: manufacturing technologies, nanotechnologies, robotics, telecommunications, physics, dental medical technologies, smart homes, speech technologies, agriculture technologies and management.

\title{
How to reference
}

In order to correctly reference this scholarly work, feel free to copy and paste the following:

Bilal Hijazi, Thomas Decourselle, Sofija Vulgarakis Minov, David Nuyttens, Frederic Cointault, Jan Pieters and Jürgen Vangeyte (2012). The Use of High-Speed Imaging Systems for Applications in Precision Agriculture, New Technologies - Trends, Innovations and Research, Prof. Constantin Volosencu (Ed.), ISBN: 978-953-510480-3, InTech, Available from: http://www.intechopen.com/books/new-technologies-trends-innovations-andresearch/the-use-of-high-speed-imaging-systems-for-applications-in-precision-agriculture

\section{INTECH}

open science | open minds

\author{
InTech Europe \\ University Campus STeP Ri \\ Slavka Krautzeka 83/A \\ 51000 Rijeka, Croatia \\ Phone: +385 (51) 770447 \\ Fax: +385 (51) 686166 \\ www.intechopen.com
}

\author{
InTech China \\ Unit 405, Office Block, Hotel Equatorial Shanghai \\ No.65, Yan An Road (West), Shanghai, 200040, China \\ 中国上海市延安西路65号上海国际贵都大饭店办公楼 405 单元 \\ Phone: +86-21-62489820 \\ Fax: +86-21-62489821
}


(C) 2012 The Author(s). Licensee IntechOpen. This is an open access article distributed under the terms of the Creative Commons Attribution 3.0 License, which permits unrestricted use, distribution, and reproduction in any medium, provided the original work is properly cited. 